\title{
Medizinisches Versorgungszentrum - geeignetes Instrument der ambulanten patientenadäquaten Versorgung und leistungsgerechten Vergütung
}

\author{
Medical Care Unit - A Suitable Instrument for Ambulatory \\ Patient-Adequate Care and Performance-Related Remuneration
}

Autoren

Institute
P. Rudolph ${ }^{1,2}$, D. Isensee ${ }^{3}$, E. Gerlach ${ }^{3}$, H. Groß ${ }^{3}$

Hochschule Magdeburg-Stendal, Fachbereich Sozial- und Gesundheitswesen, Magdeburg, Deutschland

2 EUMEDIAS Heilberufe AG, Vorstand, Magdeburg, Deutschland

3 EUMEDIAS Heilberufe AG, Consulting, Magdeburg, Deutschland
Schlüsselwörter

- Allgemeinchirurgie

- Behandlungsqualität

- Wirtschaftlichkeit

- Honorarverteilung

- Leistungserbringer

medizinisches Versorgungszentrum

Key words

- general surgery

- quality of treatment

- cost effectiveness

- fee sharing/distribution

- health care provider

- medical care unit

\section{Bibliografie}

Dol http://dx.doi.org/ $10.1055 / \mathrm{s}-0031-1283921$

Online-Publikation: 8.3.2012

Zentralbl Chir 2013; 138: 45-52

(c) Georg Thieme Verlag KG

Stuttgart · New York

ISSN 0044-409X

Korrespondenzadresse

Prof. Dr. Peter Rudolph

EUMEDIAS Heilberufe AG,

Vorstand

Hegelstraße 39

39104 Magdeburg

Deutschland

Tel.: $0391 / 5356760$

Fax: $0391 / 5356777$

Peter.Rudolph

@hs-magdeburg.de

\section{Zusammenfassung \\ $\nabla$}

Hintergrund: Die Frage, ob ein Medizinisches Versorgungszentrum ein geeignetes Instrument zur ambulanten Versorgung ist, wird seit längerer Zeit diskutiert. Ziel ist es zu untersuchen, ob MVZ ein probates Instrument der ambulanten patientenadäquaten Versorgung und leistungsgerechten Vergütung sind.

Material und Methoden: Die retro- und zum Teil prospektive Übersichtsarbeit beinhaltet Aussagen zu rechtlichen Grundlagen zum MVZ, zur Vergütung von Leistungen in MVZ, zur Entwicklung von MVZ in Deutschland und zu spezifischen Vor- und Nachteilen von MVZ. Dabei fokussiert der Artikel vordergründig allgemeingültige Fakten und ergänzt diese exemplarisch mit Beispielen für die Allgemeine, Viszeral- oder Gefäßchirurgie. Die zentralen quantitativen Daten zur MVZ-Statistik stammen aus unterschiedlichen Publikationen der Kassenärztlichen Bundesvereinigung.

Ergebnisse: Aus rechtlicher Sicht ermöglicht das Instrument MVZ ambulanten sowie stationären Leistungserbringern die Teilnahme an der Vertragsärztlichen Versorgung. Dabei erweitert sich besonders für den stationären Bereich das Spektrum an Möglichkeiten, was unter gewissen Umständen zur Sicherstellung der medizinischen Versorgung in strukturschwachen Regionen beitragen kann. Freiberufler können in erster Linie von finanzieller Risikominimierung und bürokratischer Entlastung profitieren. Die Vergütung der erbrachten Leistungen in MVZ erfolgt ana$\log \mathrm{zu}$ anderen ambulanten Leistungserbringern. Grundsätzlich sind dabei keine Nachteile, sondern vielmehr größere Gestaltungsfreiräume und Möglichkeiten zur Generierung von Zuschlägen erkennbar. Die Anzahl der MVZ in Deutschland hat sich innerhalb der letzten fünf Jahre nahezu vervierfacht, was für eine Etablierung in der ambulanten Versorgungslandschaft spricht. MVZ

\section{Abstract \\ $\nabla$}

Background: The question of whether a medical care unit is an appropriate tool for outpatient care has been discussed for a long time. Our aim is to investigate whether the MCU is an effective instrument for outpatient care and adequate performance-related remuneration.

Material and Methods: This retro- and prospective overview of the work included statements on legal foundations for medical care units, for reimbursement of services in medical care units, the development of medical care centres in Germany and a listing of the specific advantages and disadvantages of an MCU. This article focuses on the generally applicable facts and complements them with examples from general, visceral and vascular surgery. The main quantitative data on medical centre statistics come from different publications of the National Association of Statutory Health Insurance for Physicians.

Results: From a legal point of view the instrument MCU allows the participating of ambulatory and stationary care in the framework of medical care contracts. This has been especially extended for stationary applications, including the spectrum of possibilities that can contribute under certain circumstances for the provision of medical care in underdeveloped regions. Freelancers can benefit primarily from financial risk and minimising bureaucratic routine. The remuneration for services performed in the MCU is analogous to that of other ambulatory care providers. Basically, there are no disadvantages, but a greater design freedom and opportunities for the generation of aggregates are visible. The number of MCU in Germany has quadrupled in the last five years, indicating an establishment of an outpatient care landscape. MCU offers from the patient's perspective, providers and policy specific advantages and disadvantages. Indeed the benefits outweigh the disadvantages, but this is not yet verified by qualitative studies. 
bieten aus Sicht der Patienten, der Leistungserbringer und der Politik spezifische Vor- und Nachteile. Zwar überwiegen die Vorteile, jedoch sind diese bisher nicht durch qualitative Studien verifiziert.

Schlussfolgerung: Die Frage nach der Eignung von MVZ als ambulantes Versorgungsinstrument muss differenziert betrachtet werden. Unter gegenwärtigen Bedingungen erscheint das MVZ zur Sicherstellung und Ergänzung der Versorgung geeignet. Ob ein Mehrwert in der Versorgungsqualität von Patienten generiert werden kann, ist jedoch gesondert zu untersuchen, da bisher kein valides Datenmaterial vorliegt. Selbiges gilt für ökonomische Bewertungen der Kosten und Nutzen aus volkswirtschaftlicher Sicht.

\section{Einleitung}

$\nabla$

Die Überwindung der sektoralen Grenzen innerhalb der medizinischen Versorgung in Deutschland wird seit langem diskutiert. Begründet ist dieses Anliegen oftmals mit der Reduzierung von Kosten im Gesundheitswesen, der Bündelung von haus- und fachärztlicher Behandlung, der Vermeidung von Doppeluntersuchungen und der grundsätzlichen Qualitätssteigerung in der medizinischen Behandlung durch engere Kooperation von ambulanter und stationärer Versorgung. In der Vergangenheit waren diese Motive im Verbund mit der Initiierung eines verstärkten Wettbewerbs eine grundlegende Antriebskraft für die Entwicklung neuer ambulanter Organisationsstrukturen, die die Grenzen zwischen ambulantem und stationärem Bereich verringern und darüber hinaus zu vermehrter wirtschaftlicher Orientierung sowie Innovation im Bereich der medizinischen Versorgung anregen sollten. Eine daraus resultierende Versorgungstruktur ist das Medizinische Versorgungszentrum (MVZ), welches im Rahmen des GKV-Modernisierungsgesetzes (GMG) am 1.Januar 2004 eingeführt wurde [1]. Im fünften Sozialgesetzbuch (SGBV) werden im $§ 95$, der die Teilnahme an der vertragsärztlichen Versorgung regelt, MVZ als „[...] fachübergreifende ärztlich geleitete Einrichtungen, in denen Ärzte, die in das Arztregister nach Absatz 2 Satz 3 eingetragen sind, als Angestellte oder Vertragsärzte tätig sind. [...]“ definiert [2]. Als fachübergreifend gilt ein MVZ, wenn „[...] die Ärzte mit verschiedenen Facharzt- oder Schwerpunktbezeichnungen tätig sind [...]“ [2]. Seit der Einführung des MVZ sind nunmehr sieben Jahre vergangen und noch immer polarisieren MVZ als ambulantes Versorgungsinstrument [3]. Allerdings hat sich die Stellung bzw. der Anteil innerhalb der Versorgungslandschaft in Richtung einer festen Etablierung entscheidend verändert. Nach Angaben der Kassenärztlichen Bundesvereinigung (KBV) steigt die Zahl der Gründungen kontinuierlich an. Zum 1. Quartal 2006 wurden insgesamt 420 Medizinische Versorgungszentren registriert, im 1.Quartal 2010 waren es bereits 1503 MVZ [4].

Vor dem Hintergrund anhaltender Diskussionen über das Für und Wider von MVZ wird folgenden Fragestellungen nachgegangen: Wie stellt sich die rechtliche Situation für die Gründung von Medizinischen Versorgungszentren in Deutschland dar und welche Möglichkeiten bietet diese? Hat sich das MVZ in der heutigen Versorgungslandschaft etablieren können? Wie erfolgt die Vergütung der Leistungen in MVZ und ist diese leistungsgerecht? Welchen konkreten Mehrwert können MVZ zur Steigerung der Behandlungsqualität beitragen?

Nachfolgend werden die Themen rechtliche Grundlagen, Vergütung von Leistungen, Entwicklung von MVZ in Deutschland sowie Vor- und Nachteile in Bezug zu Medizinischen Versorgungs-
Conclusion: The question of the appropriateness of medical care units as outpatient care instrumentation must be considered differentially. Under current conditions it appears suitable for ensuring the MCU and the supplement of care supply. Whether a value can be generated in the quality of care of patients, however, has to be examined separately, as there are no valid data so far. The same applies to economic assessments of costs and benefits from an economic perspective.

zentren kontrovers diskutiert, um herauszufinden, ob MVZ ein geeignetes Instrument zur ambulanten medizinischen Versorgung sind.

\section{Material und Methodik \\ $\nabla$}

Um einen möglichst detaillierten und nachvollziehbaren Gesamtüberblick der aktuellen Situation der ambulanten Versorgung mithilfe von MVZ zu geben und daraus schlussfolgernd zu diskutieren, werden in dem vorliegenden retro- und zum Teil prospektiven Übersichtsartikel unterschiedliche Arten sorgfältig recherchierter Publikationen eingeschlossen. Neben der rechtlichen Situation wurden unter anderem bereits erhobene Daten zur Entwicklung von MVZ, Qualitätsindikatoren für eine patientenadäquate Versorgung und ökonomische Sachverhalte einbezogen. Grundsätzlich ist darauf hinzuweisen, dass dieser Artikel vordergründig allgemeingültige Fakten thematisiert und exemplarisch Daten für die Allgemeine, Viszeral- oder Gefäßchirurgie bereithält. Dies ist einer sehr überschaubaren Anzahl an verfügbaren Publikationen im direkten Zusammenhang mit Medizinischen Versorgungszentren geschuldet. Die zentralen Daten zur MVZ-Statistik stammen von der Kassenärztlichen Bundesvereinigung, die die jeweiligen Erhebungen der 17 Kassenärztlichen Vereinigungen bündelt und quartalsweise aufbereitet.

\section{Ergebnisse}

\section{Rechtliche Grundlagen für Medizinische Versorgungszentren}

Seit dem 1.Januar 2004 bildet $§ 95$ des SGBV die rechtliche Basis für die Teilnahme an der vertragsärztlichen Versorgung. In Absatz 1 ist festgelegt, dass zugelassene medizinische Versorgungszentren an der vertragsärztlichen Versorgung teilnehmen. In diesem Zusammenhang muss eine fachübergreifende Tätigkeit mittels unterschiedlicher Facharzt- oder Schwerpunktbezeichnungen nachgewiesen werden. Eine Einrichtung mit zwei Fachärzten für Chirurgie, die jeweils unterschiedliche Schwerpunkbezeichnungen, beispielsweise Allgemeinchirurgie und Unfallchirurgie, besitzen, ist demnach fachübergreifend. Bis Ende 2011 waren grundsätzlich alle Leistungserbringer, die aufgrund ihrer Zulassung, Ermächtigung oder vertraglichen Konstellation an der medizinischen Versorgung der gesetzlich Versicherten teilnehmen, zur Gründung eines MVZ berechtigt. In erster Linie meinte dies natürlich den klassischen Vertragsarzt, allerdings sind Plankrankenhäuser, aber auch Krankenhäuser mit existierendem Versorgungsvertrag (§108 SGBV) ausdrücklich anerkannt. Hinzu kom- 
men Vertragszahnärzte, Vertragspsychotherapeuten, ermächtigte Ärzte, Apotheken ( $\$ 129$ SGBV) und Hilfsmittelerbringer ( $\S$ 126 SGB V).

Durch die Beschränkung der Gründereigenschaft auf die im System der Gesetzlichen Krankenversicherung tätigen Leistungserbringer soll eine primär an medizinischen Vorgaben orientierte Führung des Zentrums sichergestellt werden. Nachfolgend werden die drei häufigsten Varianten betrachtet:

Variante 1: Gründer sind Vertragsärzte und / oder eine fachübergreifende Berufsausübungsgemeinschaft mit Vertragsärzten in Form einer BGB- oder Partnerschaftsgesellschaft („Freiberuflergesellschaft“). Den Versorgungsauftrag im MVZ erfüllen sowohl zugelassene Vertragsärztinnen / -ärzte als auch angestellte Ärztinnen / Ärzte.

Variante 2: Gründer sind Krankenhäuser mit einer Betreibergesellschaft (Kapitalgesellschaft), deren Gesellschafter nicht selbst die medizinische Versorgung durchführen, sondern die Versorgungsaufgaben im Rahmen der vertragsärztlichen Versorgung im MVZ mit angestellten Ärzten verwirklichen.

Variante 3: Die Gründer in einer Betreibergesellschaft setzen sich zusammen aus dem Krankenhausträger und der „Freiberuflergesellschaft“. Sie betreibt das MVZ mit angestellten Ärztinnen bzw. Ärzten sowie freiberuflichen Vertragsärzten.

Verhältnismäßigkeiten bei der Verteilung der Gesellschafteranteile wurden vom Gesetzgeber nicht konkret thematisiert, wodurch alle Konstellationen von zugelassenen Gründern denkbar waren. Die potenziellen Gründer eines MVZ konnten sich nach altem Recht für jede gängige Gesellschaftsform entscheiden. Allerdings wurden mit der Einführung des „Gesetzes zur Verbesserung der Versorgungsstrukturen in der gesetzlichen Krankenversicherung" (GKV - Versorgungsstrukturgesetz - GKV-VStG), welches zum 01.01.2012 in Kraft trat, einige wichtige Veränderungen eingeführt. Betroffen ist unter anderem $§ 95$ SGBV, der die Teilnahme an der vertragsärztlichen Tätigkeit und damit die Gründung sowie Zulassung von MVZ regelt. Seit dem 1.Januar 2012 können MVZ nur noch von zugelassenen Ärzten und Krankenhäusern, Erbringern nichtärztlicher Dialyseleistungen sowie von gemeinnützigen Trägern, die aufgrund von Zulassung oder Ermächtigung zur Teilnahme an der vertragsärztlichen Versorgung berechtigt sind, gegründet werden. Die Auswahlmöglichkeit der Rechtsform wurde auf Personengesellschaften, Gesellschaften mit beschränkter Haftung und Genossenschaften reduziert. Grundsätzlich gilt jedoch, dass bei der Wahl der Rechtsform die Normen des Berufsrechts Beachtung finden sollten. Im Fall von Ärzten ist dies die Berufsordnung, die beispielsweise die Gründung einer Handelsgesellschaft untersagt, da der ärztliche Beruf kein eigenständiges Gewerbe darstellt. Die Leitung eines MVZ orientiert sich an der medizinischen Letztverantwortlichkeit. In diesem Kontext ist das Szenario der ärztlichen Gesamtleitung neben einer Trennung der Verantwortlichkeiten von medizinischen und wirtschaftlichen Kompetenzen möglich. Demnach muss die medizinische Leitung definitiv in ärztlicher Hand liegen, wobei besondere Qualifikationen oder die Voraussetzung einer Tätigkeit im MVZ bis Ende 2011 nicht konkret gefordert waren. Ein ärztlicher Leiter musste nicht zwangsläufig im Medizinischen Versorgungszentrum als Leistungserbringer tätig sein oder zu den Gesellschaftern gehören. Allerdings wurden auch hier im GKV-VStG Änderungen vorgenommen. In Absatz 1 wurde der Satz „Der Ärztliche Leiter muss in dem medizinischen Versorgungszentrum selbst als angestellter Arzt oder als Vertragsarzt tätig sein; er ist in medizinischen Fragen weisungsfrei." eingefügt. Für alle MVZ, die bis zum Inkrafttreten des GKV-VStG be- reits zugelassen waren, gilt ein sechsmonatiger Bestandsschutz, in dem auf die jeweiligen neuen Regelungen umgestellt werden kann. Sollten die Gründungsvoraussetzungen und die Bestimmungen der ärztlichen Leitung innerhalb der Frist nicht erfüllt werden, ist dem jeweiligen MVZ die Zulassung zu entziehen [2, 5-10].

Das Versorgungsstrukturgesetz ist trotz vieler kritischer Meinungen in Kraft getreten. Letztendlich bleibt abzuwarten, wie es in der Praxis umgesetzt wird [11].

Schlussfolgernd stellt das MVZ eine Möglichkeit für stationäre Leistungserbringer und deren Träger dar, in größerem Umfang als es beispielsweise über Ermächtigungen möglich ist, an der ambulanten Versorgung teilzunehmen. Dies ist vor allem vor dem Hintergrund der Sicherung der medizinischen Versorgung in strukturschwachen Regionen von Bedeutung, da dort oftmals die Motivation zur Niederlassung einzelner Ärzte nicht gegeben ist. Von dieser stabilisierenden Ergänzung in der ambulanten Versorgungslandschaft profitiert nicht zuletzt der Patient. Zwar können mit den MVZ-Gründungen auf längere Sicht Einbußen in der Wohnortnähe der ambulanten Leistungserbringung einhergehen, dennoch kann auf diese Weise eine fachübergreifende ambulante Patientenversorgung im strukturschwachen ländlichen Raum gesichert werden. Dies resultiert nicht zuletzt daraus, dass auch ambulanten Leistungserbringern eine Möglichkeit zur Niederlassung als MVZ eingeräumt wird, die sich insbesondere durch eine Verteilung des finanziellen Risikos auf mehrere Ärzte auszeichnet. Zusätzlich ist das MVZ die einzige Versorgungsform, welche eine medizinische Behandlung mit ausschließlich angestellten Ärzten ermöglicht.

\section{Vergütung der Leistungen}

Grundsätzlich erfolgt die Honorarverteilung für MVZ analog zur Honorierung anderer ambulanter Leistungserbringer. Demnach erhält jeder Leistungserbringer im MVZ ein gesondertes Regelleistungsvolumen (RLV) und ein Qualifikationsgebundenes Zusatzvolumen (QZV). Die Summe aus diesen Volumina ergibt das Budget für Kassenärztliche Leistungen des MVZ. Darüber hinaus sind freie Leistungen, Einzelleistungen nach Einheitlichem Bewertungsmaßstab (EBM) bzw. privatärztliche Leistungen (z.B. IGeL) gemäß der Gebührenordnung für Ärzte (GOÄ) abrechenbar. Ergänzt wird dieses Spektrum durch IV-Verträge, BG-Leistungen als D-Arzt sowie Gutachten. Die Besonderheit im MVZ oder ähnlichen Versorgungsstrukturen liegt insbesondere in der Möglichkeit, nicht ausgeschöpfte Volumina untereinander zu verrechnen. Hat beispielsweise Chirurg A sein Budget nicht ausgelastet, kann Chirurg B die Differenz in sein Honorarbudget einfließen lassen und demnach mehr Leistungen erbringen. In diesem Zusammenhang ist jedoch darauf zu verweisen, dass gegenüber der klassischen Einzelpraxis nicht jeder Arzt-Patient-Kontakt gleichzeitig einen Behandlungsfall darstellt. In Berufsausübungsgemeinschaften wie dem MVZ wird zwischen Behandlungs- und Arztfällen unterschieden, da Überweisungen innerhalb des MVZ nicht zulässig sind. Ein Behandlungsfall kann somit aus mehreren Arztfällen bestehen, wenn mehrere Leistungserbringer an der Behandlung eines Patienten beteiligt sind. Da die Fallzahlen (Behandlungsfälle) für die Bestimmung der jeweiligen Volumina entscheidend sind, muss dieser Fakt unbedingt berücksichtigt werden. Darüber hinaus besteht eine weitere Besonderheit bei der Bestimmung der Volumina, die im direkten Zusammenhang mit der Thematik der Arzt- bzw. Behandlungsfälle steht. Fach- 
oder schwerpunktübergreifende BAG oder MVZ erhalten Zuschläge auf das bestehende RLV in Abhängigkeit vom Kooperationsgrad. Ab dem 3. Quartal 2011 wird auf Grundlage eines Beschlusses des Bewertungsausschusses der KBV vom 25.Januar 2011 der Kooperationsgrad nach der tatsächlichen Kooperation der Ärzte berechnet. Vor dem 3. Quartal konnten MVZ je nach Anzahl der beteiligten Fach- bzw. Schwerpunktgruppen zwischen $10 \%$ und $40 \%$ Zuschlag auf das Gesamt-RLV des MVZ beanspruchen. Die Neuregelung bewirkt eine Festsetzung des Zuschlages anhand der tatsächlichen Arzt- und Behandlungsfälle. Der Kooperationsgrad der Ärzte wird vom Bewertungsausschuss wie folgt definiert:

Kooperationsgrad $=[$ (Summe Arztfälle im Vorjahresquartal $\div$ Summe Behandlungsfälle im Vorjahresquartal) -1] ×100

Für die Leistungserbringer bedeutet dies einen Zuschlag in Abhängigkeit vom Kooperationsgrad auf das RLV, soweit dieser Kooperationsgrad mindestens $10 \%$ beträgt. Die Zuschläge werden stufenweise in 5\%-Schritten erhöht, wobei die Obergrenze weiterhin bei maximal $40 \%$ liegt. Der dargestellte Sachverhalt ist zum besseren Verständnis in $\bullet$ Abb. 1 visualisiert [2, 12].

Die Zuweisung der Einnahmen innerhalb des MVZ wird über individuelle Vereinbarungen und Verträge geregelt und sichergestellt, wobei die Verteilung der Honoraranteile den tatsächlichen Leistungsanteilen der beteiligten Ärzte entsprechen kann.

Grundsätzlich ist festzuhalten, dass die Leistungsabrechnung nach dem in MVZ Anwendung findenden Vergütungssystem keinerlei Nachteile für die dort tätigen Ärzte birgt. Das Funktionsprinzip des MVZ gestattet einen Mehrgewinn an Abrechnungsund Handlungsspielräumen: Zum einen können durch die Möglichkeit der Arbeitsteilung und den daraus resultierenden zeitlichen Ressourcen auch Zusatzleistungen (z. B. IGeL) erbracht werden, für die sonst weniger Zeit zur Verfügung steht. Zum anderen ist die Möglichkeit der arztübergreifenden Verrechenbarkeit von ungenutzten Volumina herauszustellen. Hinzukommend können die dort tätigen Ärzte von möglichen Zuschlägen, die für den jeweiligen Kooperationsgrad gewährt werden, profitieren und das ihnen zugewiesene Honorar aufwerten. Aus Patientensicht kann aus diesen Spielräumen eine adäquate Behandlung resultieren, da den Leistungserbringern mehr Möglichkeiten gegeben sind, den systembedingten Schwierigkeiten der Budgetierung zu begegnen. Aus chirurgischer Sicht könnten beispielsweise die Ärzte ihre eigenen Patienten prä- und postoperativ im MVZ behandeln und den operativen Eingriff selbstständig im Krankenhaus als Angestellter oder im Rahmen eines IV-Vertrags durchführen. Zusätzlich können neben der Ausschöpfung der durch die KV zugewiesenen RLV bzw. QZV BG-Leistungen erbracht und Gutachten erstellt werden.

\section{Entwicklung von Medizinischen Versorgungszentren in Deutschland \\ $\nabla$}

Als am 1.Januar 2004 mit dem GKV-Modernisierungsgesetz (GMG) die Möglichkeit zur Gründung eines MVZ geschaffen wurde, war nicht absehbar, ob und in welchem Ausmaß sich dieses neue Versorgungsmodell etablieren würde. Abb. 2 zeigt in diesem Zusammenhang die Entwicklung der MVZ in Deutschland auf. Im 1.Quartal 2005 gab es 121 als Leistungserbringer eingetragene MVZ $[13,14]$. In den Folgejahren haben sich jeweils zum ersten Quartal eines Jahres relativ konstante Wachstumsraten er-

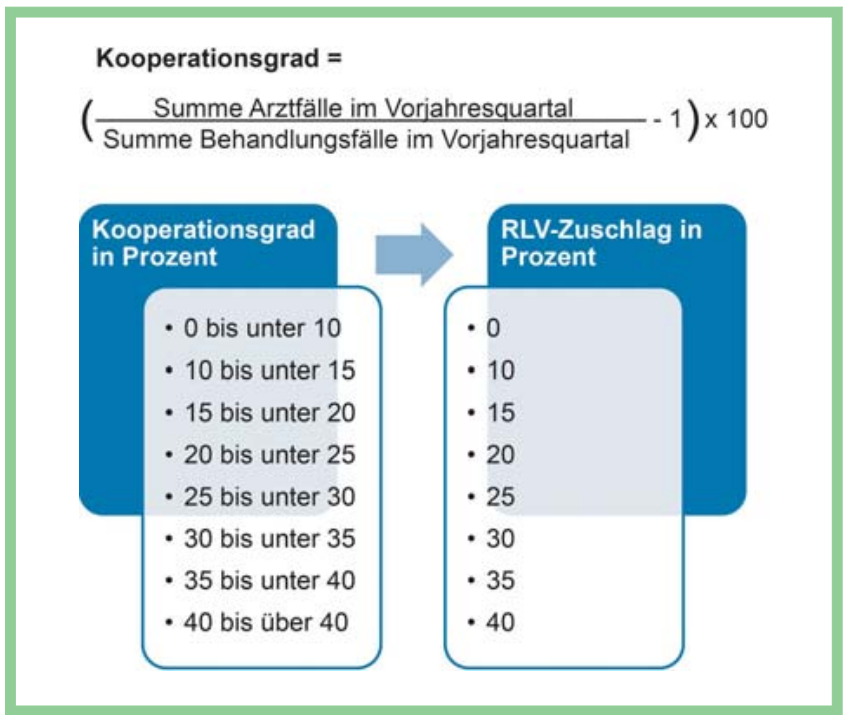

Abb. 1 Bestimmung des Kooperationsgrades (Quelle: Eigene Darstellung).

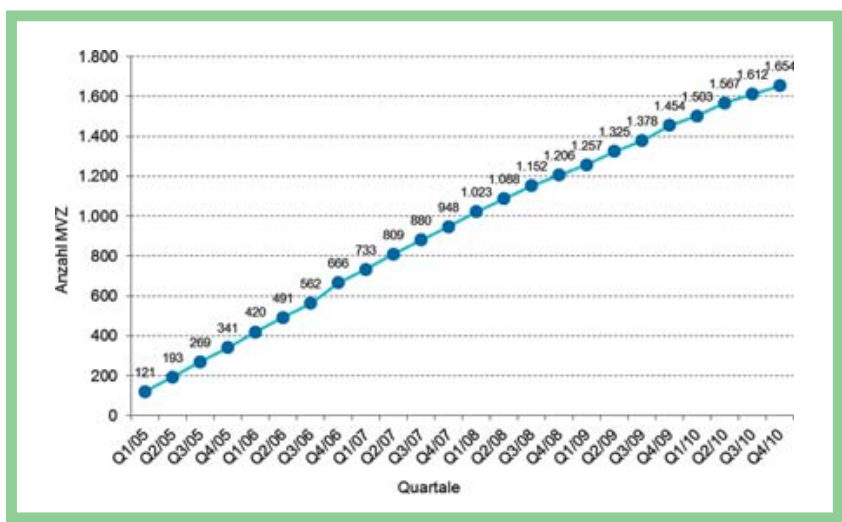

Abb. 2 Entwicklung der MVZ-Anzahl (Quelle: Eigene Darstellung).

geben, die im Schnitt auf 200 bis 300 Neugründungen pro Jahr zurückzuführen waren. Wurden 2006 insgesamt $420 \mathrm{MVZ}$ registriert, waren es 2007 733, ehe zum 1. Quartal 2008 die Marke von 1000 mit insgesamt $1023 \mathrm{MVZ}$ durchbrochen wurde. 2009 erfasste die KBV 1257 Zentren, 2010 wurden in Deutschland 1503 MVZ notiert $[4,15,16]$. Im selben Jahr stieg die Zahl der MVZ weiterhin kontinuierlich an. Die aktuellsten Zahlen liegen aus dem 4.Quartal 2010 vor. Zum Stichtag 31.Dezember 2010 registrierte die KBV 1654 MVZ $[17,18]$. Bei den Gründungen von MVZ ist demnach keine Stagnation ersichtlich und zudem nicht zu erwarten.

Ein weiterer Trend ist bei der Art der Trägerschaft ersichtlich. Prinzipiell kann vereinfacht von zwei Modellen ausgegangen werden: MVZ in vertragsärztlicher Trägerschaft und MVZ in Krankenhausträgerschaft. Bundesweit stellt die erste Variante die verbreitetere Methode dar, jedoch ist mit zunehmender zeitlicher Etablierung von MVZ ein steigender Anteil an Krankenhaus-MVZ ersichtlich. In $\bullet$ Abb. 3 wird dieser Sachverhalt mithilfe der prozentualen Anteile der Krankenhaus-MVZ an allen MVZ aufgezeigt. Demnach waren im 1.Quartal 2006 von insgesamt $420 \mathrm{MVZ} 118$ Einrichtungen an Krankenhäuser angebunden. Im 4. Quartal 2010 waren bereits 647 Krankenhaus-MVZ im Vergleich zu 1654 Gesamt-MVZ vertreten. Im Diagramm wird allerdings auch eine Stabilität des Anteils ab dem 1. Quartal 2009 deutlich. Zusätzlich muss jedoch erwähnt werden, dass die Krankenhaus-MVZ vor allem in den neuen Bundesländern einen 


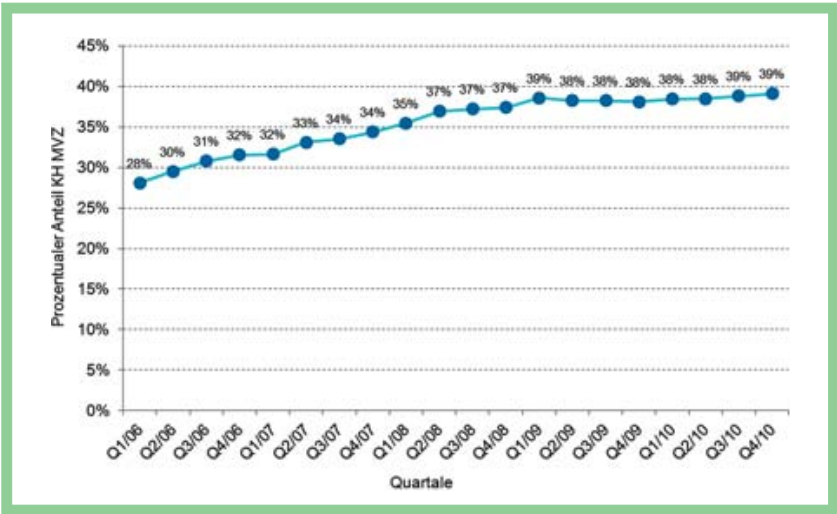

Abb.3 Entwicklung des prozentualen Anteils von Krankenhaus-MVZ (Quelle: Eigene Darstellung).

größeren Zulauf besitzen, während in den alten Bundesländern von Ärzten gegründete MVZ dominieren [4, 18-20].

Die umfassende Beurteilung der MVZ-Entwicklung bedarf auch einer genaueren Betrachtung der Arbeitsgröße. Wird diese anhand der Anzahl der in einem MVZ tätigen Ärzte gemessen, zeigt sich ein differenzierter Trend auf. Im Gegensatz zur permanent steigenden Anzahl von MVZ zeigt sich die durchschnittliche Anzahl der dort tätigen Ärzte zunächst relativ stabil. In $\odot$ Abb. 4 ist erkennbar, dass beispielsweise im 1.Quartal 2006 durchschnittlich 3,9 Ärzte in MVZ arbeiteten. Im 4. Quartal 2010 waren durchschnittlich 5,2 Ärzte in MVZ tätig. Nominell ist somit ein eher geringer Anstieg feststellbar. Wird hingegen die Wachstumsrate betrachtet, ist innerhalb dieser viereinhalb Jahre eine Steigerung von ca. $25 \%$ zu verzeichnen $[4,20]$.

Die Analyse verschiedener Entwicklungsparameter legt den Schluss nahe, dass sich das MVZ als neue Organisationsform der ambulanten Leistungserbringung in der Versorgungslandschaft etabliert hat. Seit 2004 sind sowohl kontinuierliche Zuwachsraten als auch Anstiege bei den absoluten MVZ-Zahlen zu verzeichnen. So bieten MVZ aufgrund der Risikoverteilung eine weit größere finanzielle Sicherheit als es andere ambulante Versorgungsformen vermögen. Sowohl dieser Sachverhalt als auch die Zunahme von MVZ in stationärer Trägerschaft leisten einen entscheidenden Beitrag zur nachhaltigen Sicherstellung der ambulanten Versorgung in strukturschwachen und von Unterversorgung bedrohten Regionen. Der Anstieg der durchschnittlichen Arbeitsgröße von MVZ ist ein Indikator für die Attraktivität einer Tätigkeit in MVZ und somit auch für die Zukunftsfähigkeit dieser Versorgungsform.

\section{MVZ mit chirurgischem Anteil \\ $\nabla$}

Stellvertretend für den Anteil chirurgischer Beteiligung in Medizinischen Versorgungszentren wird das 4. Quartal 2010 analysiert. In den bereits erwähnten $1654 \mathrm{MVZ}$ waren insgesamt 8610 Ärzte tätig. Im Vergleich der Anteile der jeweiligen Fachgruppen befinden sich die Chirurgen mit 636 Ärzten $(7,4 \%)$ in $345 \mathrm{MVZ}(20,9 \%)$ an dritter Stelle hinter den Hausärzten mit 1303 Ärzten (15,1\%) in 687 MVZ (41,5\%) sowie den Internisten mit 871 Ärzten (10,1\%) in 435 MVZ (26,3\%) [19]. o Abb. 5 visualisiert den Anteil der MVZ mit chirurgischer Beteiligung (20,9\%) und deren Zusammensetzung. Daraus resultiert ein Anteil von 35,4\% an Fachärzten für Chirurgie im durchschnittlichen MVZ, in dem Chirurgen tätig sind. Grundsätzlich ist die Fachgruppe

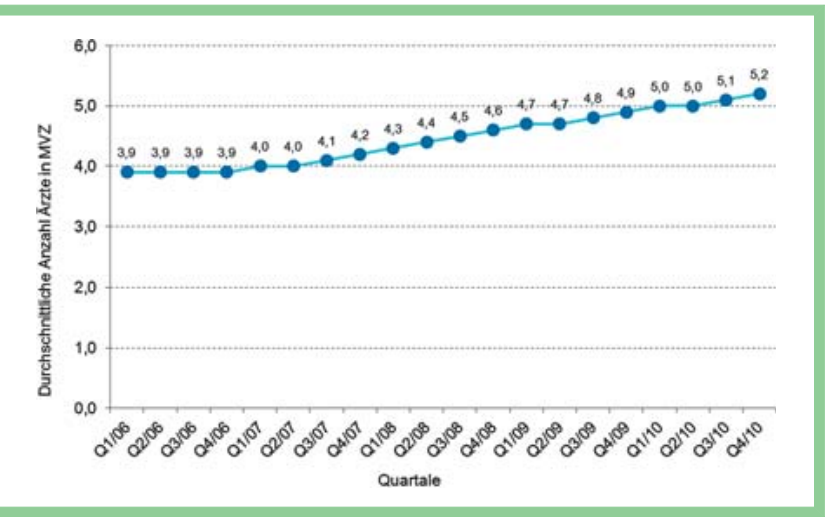

Abb.4 Entwicklung der Arbeitsgröße von MVZ (Quelle: Eigene Darstellung).

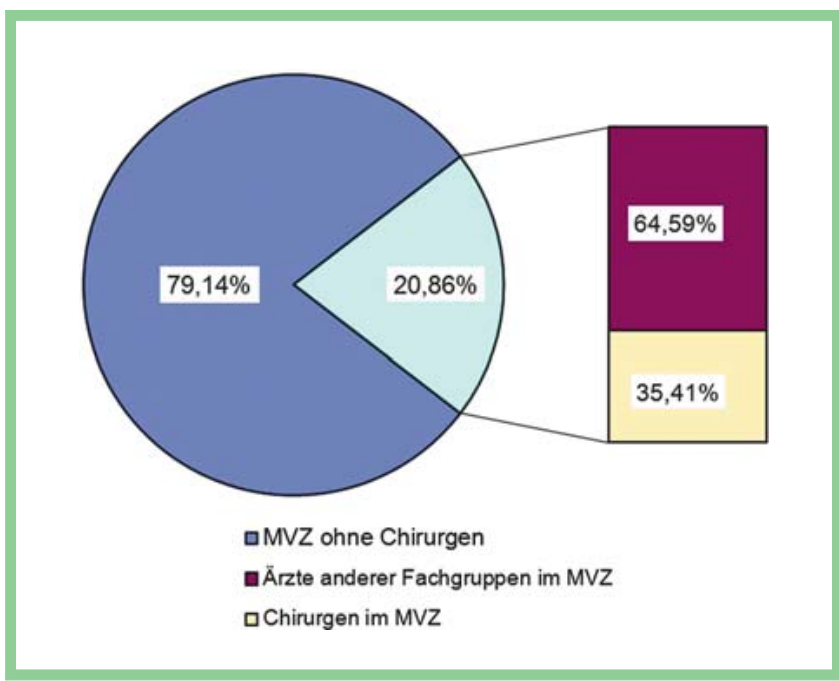

Abb.5 MVZ mit chirurgischer Beteiligung im 4. Quartal 2010 (Quelle: Eigene Darstellung).

der Chirurgen relativ stark und zugleich konstant vertreten. Dies lässt sich am Anteil von Chirurgen an den Gesamtzahlen der in MVZ tätigen Ärzte seit Einführung der MVZ im 1. Quartal 2005 bis zum 4. Quartal 2010 belegen, der kontinuierlich zwischen 7\% und $8 \%$ liegt $[14,15,18,19]$.

Die generellen Schlussfolgerungen anhand der Entwicklung von MVZ können auch auf MVZ mit chirurgischer Beteiligung übertragen werden.

\section{Vor- und Nachteile Medizinischer Versorgungszentren} $\nabla$

Wie jede Versorgungsform weisen MVZ ebenfalls spezifische Vorund Nachteile auf. An dieser Stelle sollen stellvertretend einige der Punkte aus $\boldsymbol{O}$ Abb. 6 thematisiert werden. Dabei lassen sich vier verschiedene Perspektiven differenzieren, die sich an Patienten, Ärzten, Krankenhäusern und der Gesundheitspolitik ausrichten. Aus Sicht der Patienten liegen Vorteile durchaus in einem größeren Angebot im Leistungsspektrum unter einem Dach, längeren Sprechstunden- und verkürzten Wartezeiten durch vielfältigere organisatorische Möglichkeiten und die Vermeidung von Doppeluntersuchungen. Allerdings sind aufgrund einer geringeren Flächenabdeckung durch MVZ durchaus längere Anfahrtswege denkbar. Wird die Perspektive der Ärzte betrachtet, sind 


\section{Vorteile von MVZ}
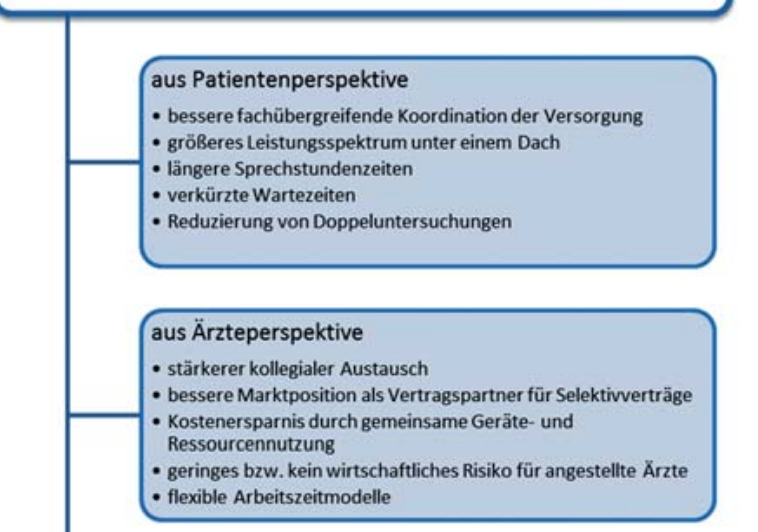

aus Krankenhausperspektive

- Kostenersparnis durch Verlagerung von vor-und nachstationären Leistungen an das MVZ

- zusätzliche Einnahmen aus dem ambulanten Bereich

- Generierung zusätzlicher Einweisungen durch das eigene MVZ

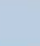

- ggf. höhere Qualitāt durch engere Verzahnung zwischen hausund fachärztlicher Versorgung sowie zwischen ambulanter und stationärer Versorgung

- ggf. höhere Wirtschaftlichkeit durch Kosteneinsparungen

\section{Nachteile von MVZ}

mögliche Kostenersparnisse durch gemeinsame Ressourcennutzung sowie stärkerer kollegialer Austausch mit teamorientierten Arbeitsweisen positiv herauszustellen. Mit Letzterem ist jedoch ein größerer interner Kommunikations- und Koordinationsaufwand verbunden. Die Aufgabe der Freiberuflichkeit zugunsten einer Anstellung wird häufig als negativer Aspekt empfunden. Durch vielfältige Modelle, wie beispielsweise gleichzeitige bzw. ergänzende Anstellung in einem Krankenhaus mit Fokus auf operative Tätigkeiten (speziell Chirurgie), kann dieser Aspekt auch als vorteilhaft empfunden werden. Zusätzlich stehen dem vermeintlich negativen Aspekt geringere wirtschaftliche Risiken für den angestellten Arzt positiv gegenüber.Die Krankenhausperspektive verbucht bei den Vorteilen die Möglichkeit zur Generierung zusätzlicher Einnahmen aus dem ambulanten Bereich. Als ein weiterer ambulanter Leistungserbringer sollte der Kontakt zu den anderen niedergelassenen Einweisern kooperativ gestaltet werden, da diese bei zu starkem Konkurrenzempfinden mit verändertem Ein- bzw. Überweisungsverhalten reagieren können. Pro MVZ sprechen aus gesundheitspolitischer Sicht vor allem die Möglichkeit einer engeren Verzahnung von haus- und fachärztlicher Versorgung sowie der Abbau der sektoralen Grenzen zwischen ambulanter und stationärer Behandlung. Nachteilig sind mögliche Forderungen an den angestellten Arzt, Patienten in erster Linie an den stationären Träger zu überweisen, was den optimalen Behandlungsverlauf beeinträchtigen kann. Eine Reduzierung der Wohnortnähe durch die räumliche Konzentration von Ärztinnen und Ärzten ist ein weiterer Nachteil aus gesundheitspolitischer Perspektive [21].

Nachfolgend werden einige praktische Beispiele stellvertretend für mögliche Vorzüge von MVZ aufgezeigt. Einen Einstieg bildet dabei der sich abzeichnende Nachwuchsmangel in der Chirurgie. Knapp mehr als die Hälfte der Belegschaft in der Chirurgie ist älter als 50 Jahre und nur wenige der Medizinabsolventen sehen ihre Zukunft in diesem Fachgebiet. Als Gründe werden häufig Defizite in der Weiterbildung, mangelnde Karriereperspektiven, Gehaltsfragen oder die Work-Life-Balance aufgeführt. Diese könnten auch auf andere medizinische Fachgebiete übertragen werden, jedoch ist es gerade die Chirurgie, die nach bestmöglicher praktischer Ausbildung und guter Organisation verlangt [22]. In diesem Zusammenhang können Medizinische Versorgungszentren neue Ansätze bieten, um die Attraktivität des Chirurgenberufs zu steigern. Durch die ambulanten chirurgischen Sprechstunden wird eine selbstständige Behandlung von Patienten ermöglicht. Somit entstehen Freiräume für eigenständige Erfahrungen. In Anbetracht des anvisierten Abbaus der sektoralen Grenzen sind dadurch Ausbildungsinhalte für stationäre als auch ambulante Aufgaben möglich. Darüber hinaus bieten sich neue Gehaltsmodelle durch Umsatzbeteiligungen an. Arbeits- 
verträge, die stationäre sowie ambulante Bereiche kombinieren, verhelfen zu Spielräumen zur Selbstverwirklichung und können flexiblere Arbeitszeiten begünstigen.

Als weiterer Vorteil kann eine Qualitätssteigerung im Bereich der prä- und postoperativen Untersuchungen mit bildgebenden Verfahren gesehen werden. Durch die gemeinsame Ressourcennutzung ist es einem MVZ eher möglich, durch aktuelle Gerätegenerationen in der Sonografie und beim Röntgen deutliche Verbesserungen in den Erkennungsraten zu erreichen. So besteht die Möglichkeit, bereits im ambulanten Bereich eine deutlich bessere präoperative Diagnosesicherung durch einen erfahrenen Untersucher durchzuführen. Beispielhaft kann hier die akute Appendizitis aufgeführt werden [23]. Ebenso bestehen bei postoperativen Untersuchungen und Routinekontrollen Möglichkeiten, durch neue Verfahren wie die kontrastmittelverstärkte Sonografie in der Nachsorge im ambulanten Bereich stationsersetzende Behandlungsschemata mit geringerer Strahlenbelastung für den Patienten als auch geringeren Gesamtkosten zu etablieren. So kann z.B. ein großer Teil der Routinenachkontrollen nach endovaskulärer Versorgung, wie im Nachsorgeschema des Universitätsklinikums Regensburg beschrieben, im MVZ stattfinden [24].

Am Beispiel der Erfassung von Nachsorgedaten bei Brustkrebspatientinnen lässt sich ebenfalls ein Vorteil festmachen. So gestaltet sich eine vollständige Erfassung des weiteren Krankheitsund Behandlungsverlaufs bei Tumorpatienten in einem reinen Klinikregister als schwierig. Da die Nachsorge in der Regel außerhalb der behandelnden Klinik stattfindet und keine einheitlichen Dokumentationsvorlagen verwendet werden, ist ein hoher Prozentsatz an vollständigen Informationen unrealistisch. Abhilfe können in diesem Fall MVZ schaffen, die mit dem klinischen Bereich eng verzahnt sind [25]. Demnach werden einheitliche Dokumentationsstandards verwendet und die Nachsorge ist an die speziellen Bedürfnisse des Patienten angepasst. Denkbar sind daraus entstehende regionale Patientenregister, welche die Vollständigkeit der benötigten Informationen wahrscheinlicher machen.

Insgesamt überwiegen die Vorteile, welche durch Medizinische Versorgungszentren entstehen können. Allerdings obliegt es den einzelnen Trägern, Leistungserbringern und Patienten, von diesen bestmöglich zu partizipieren. Zusätzlich gilt es, Nachteile mittels gezielter politischer Entscheidungen, transparenter Kommunikation, gut durchdachter Aufbau- und Ablauforganisation und den richtigen Motivationsanreizen bereits im Vorhinein zu beseitigen.

Grundsätzlich stellt sich die Frage, ob die Qualität der medizinischen Versorgung verbessert werden kann. Dabei kann eine optimale Versorgungsqualität als gesichert gelten, wenn sie zur richtigen Zeit, am richtigen Ort und in adäquater Art und Weise erbracht wird. Je nach Organisationsform setzt dies unterschiedliche Anforderungen an interner und externer Kooperation sowie Koordination voraus. In $\mathbf{0}$ Abb.7 wird ersichtlich, dass MVZ in Summe den geringsten Kooperations- und Koordinationsaufwand benötigen, um ein umfassendes Spektrum an notwendigen medizinischen Leistungen vorhalten zu können. Exemplarisch weisen Einzelpraxen einen hohen externen Abstimmungsaufwand zur Vernetzung fachärztlicher Kompetenzen auf, wohingegen MVZ eine größere Bandbreite an Leistungen unter einem Dach anbieten. Gleichzeitig ist der interne Organisationsaufwand aufgrund der kleineren Arbeitsgröße von MVZ im Vergleich zu integrierten Vollversorgungssystemen gering. Schlussfolgernd kann im Idealfall mehr Zeit für die bedarfsgerechte medizinische Versorgung von Patienten aufgebracht werden [26].

\section{Fazit \\ $\nabla$}

Grundsätzlich kann festgestellt werden, dass die Frage nach der Eignung von MVZ als ambulantes Versorgungsinstrument differenziert zu betrachten ist. Ausgehend von den zentralen Fragestellungen bietet die rechtliche Situation für die Gründung von MVZ eine Variation an Möglichkeiten für stationäre sowie ambulante Leistungserbringer. Insbesondere für stationäre Träger ergibt sich mit der Gründung eines MVZ, welches auf das Leistungsprofil des Krankenhauses abgestimmt ist, neben der Generierung neuer Patientenströme die Möglichkeit, sich verstärkt als hochspezialisierter Leistungserbringer in der ambulanten Versorgungslandschaft zu etablieren. Zugleich profitieren die Gesellschafter eines MVZ in freiberuflicher Trägerschaft von einer damit einhergehenden Risikoteilung in finanzieller Hinsicht, den aus der gemeinsamen Nutzung der Räumlichkeiten, des Personals und der Medizintechnik resultierenden Kostenersparnissen

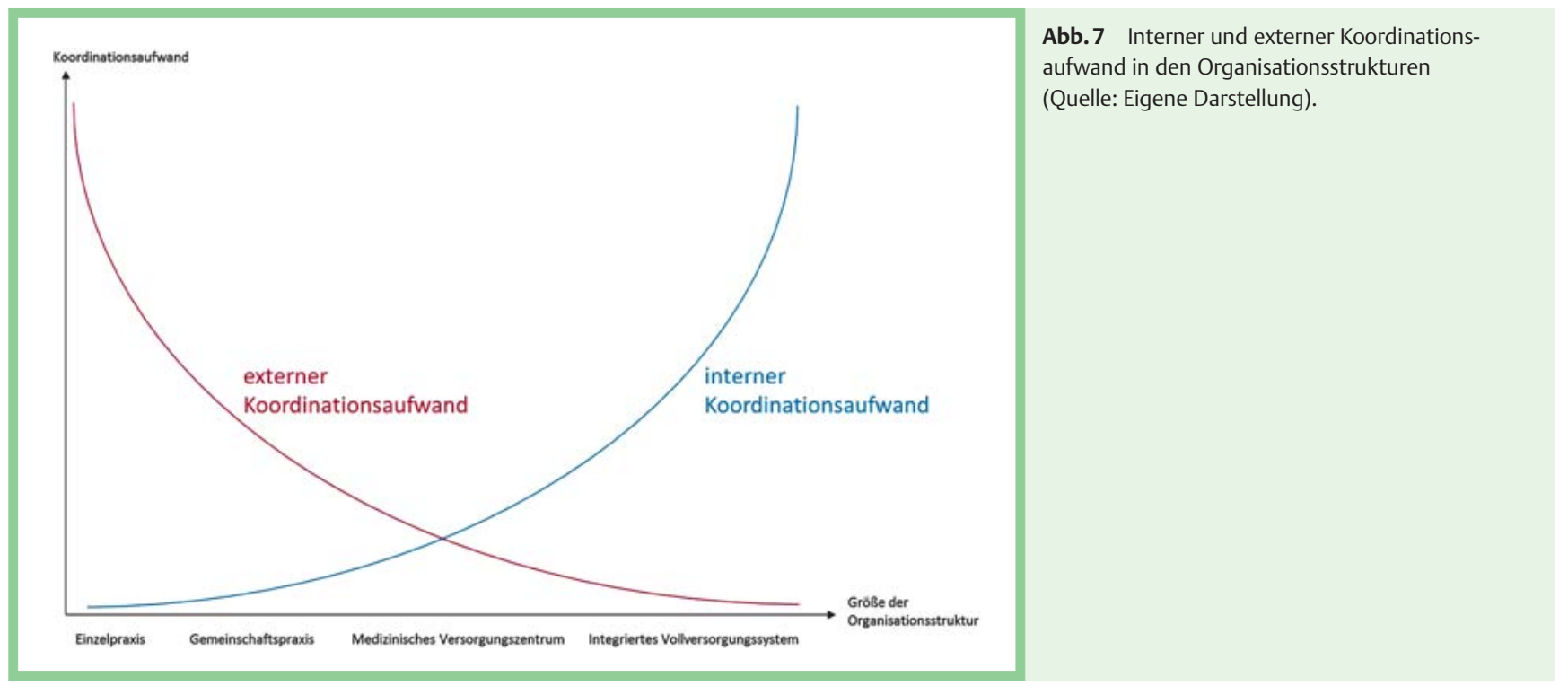


sowie den reduzierten bürokratischen Anforderungen an den einzelnen Arzt.

Werden die Zuwachsraten bzw. die absolute MVZ-Anzahl betrachtet, so erscheint das Instrument unter gegenwärtigen Bedingungen als etabliert. Während MVZ in Ballungszentren als oftmals hochspezialisierte Facheinrichtungen die ambulante Versorgung erweitern, tragen sie im ländlichen Raum eher zur Minderung einer drohenden Unterversorgung bei. Grundsätzlich sollten MVZ jedoch nicht als konkurrierendes Instrument wahrgenommen, sondern vielmehr als Ergänzung zum bestehenden Spektrum an Leistungserbringern verstanden werden. So geht Bernd Köppl, Vorsitzender des Bundesverbandes für Medizinische Versorgungszentren-Gesundheitszentren-Integrierte Versorgung e.V. (BMVZ), davon aus, dass der bundesweite Anteil von MVZ an der ambulanten Versorgung inzwischen $4 \%$ bis $5 \%$ beträgt [21].

In Anbetracht einer leistungsgerechten Vergütung können für das Instrument MVZ im Gesamten sowie für die darin tätigen Ärzte, ob angestellt oder freiberuflich tätig, im Vergleich zu anderen Versorgungsformen keine Nachteile festgestellt werden. Viel eher profitieren die Gesellschafter im Hinblick auf das Betriebsergebnis u.a. von flexiblen Verrechnungsmöglichkeiten der einzelnen Leistungsvolumina sowie den Ressourcen zur Erbringung von zusätzlichen Leistungen (z.B. D-Arzt-Modell oder Erstellung von Gutachten). Zusätzlich weist die steigende Arbeitsgröße auf die Attraktivität einer Anstellung in MVZ hin. So entspricht das Fixgehalt von Ärzten im Angestelltenverhältnis häufig dem Durchschnittshonorar der Fachgruppe in der Niederlassung. Zusätzlich besteht die Option, dieses Fixgehalt über leistungsorientierte Gehaltsbestandteile aufzuwerten. Diese Leistungsorientierung kann zur Steigerung der Behandlungsqualität in MVZ beitragen.

Ähnliche Effekte werden durch eine Verringerung des bürokratischen Aufwands und die damit einhergehende Rückbesinnung auf die medizinische Tätigkeit möglich. Weitere Ressourcen zur Generierung eines Mehrwertes in der medizinischen Behandlung, wie sie zuvor unter den potenziellen Vorteilen eines MVZ beschrieben wurden, können durch optimierte Kooperationsund Koordinationsstrukturen freigesetzt werden.

Somit ist das Instrument MVZ als Ergänzung zu den bestehenden Versorgungsstrukturen im Hinblick auf die Sicherstellung der ambulanten Versorgung geeignet. Inwieweit mit dieser Versorgungsform jedoch ein qualitativer Mehrwert für die Patienten tatsächlich generiert werden kann, ist gesondert zu untersuchen. Bis dato liegt hierzu wenig publiziertes Datenmaterial vor. Selbiges gilt für ökonomische Bewertung von Kosten und Nutzen aus volkswirtschaftlicher Sicht. Daher ist es empfehlenswert, die sich ergebenden Fragestellungen in den Fokus der weiteren Versorgungsforschung zum MVZ zu stellen.

\section{Interessenkonflikt: Nein}

\section{Literatur}

1 Bundesärztekammer (BÄK). Medizinische Versorgungszentren (26.07. 2006) Im Internet: www.bundesaerztekammer.de Stand: 01.04.2011

2 Bundesministerium der Justiz. Sozialgesetzbuch (SGB) Fünftes Buch (V) Gesetzliche Krankenversicherung (24.03.2011). Im Internet: www. gesetze.juris.de Stand: 01.04.2011

3 Ewig E, Voigt B. Medizinisches Versorgungszentrum: verherrlicht und verteufelt. Z Psychosom Med Psychother 2006; 52: 210-218
4 Kassenärztliche Bundesvereinigung (KBV). Entwicklung der Medizinischen Versorgungszentren (31.03.2010). Im Internet: www.kbv.de Stand: 14.03.2011

5 Münzel H, Zeiler N. Ambulante Leistungen in und an Krankenhäusern. Grundlagen und praktische Umsetzungen. 1. Aufl. Stuttgart: Kohlhammer; 2009

6 Kasper J. Medizinische Versorgungszentren. Rechtliche Grundlagen. Praxis Wissen Medizinische Versorgungszentren 2007; 1: 9-15

7 Schmitz U, Dieck J, Crespin S, Riedel R. Die Abrechnung medizinischer Leistungen in Medizinischen Versorgungszentren und anderen Formen kooperativer Berufsausübung unter Berücksichtigung der EBMReformen. Köln: Rheinische Fachhochschule Köln; 2008; 1. Aufl.

8 AOK-Bundesverband. Aktuelle Gesetzgebung. Im Internet: www.aokgesundheitspartner.de Stand: 12.08.2011

9 Bundesministerium für Gesundheit. Entwurf eines Gesetzes zur Verbesserung der Versorgungsstrukturen in der gesetzlichen Krankenversicherung. Im Internet: www.bmg.bund.de Stand: 27.07.2011

10 Bundesministerium für Gesundheit. Unterrichtung durch die Bundesregierung (17/7274). Stellungnahme des Bundesrates und Gegenäußerung der Bundesregierung vom 05.10.2011. Berlin: Bundesanzeiger Verlagsgesellschaft mbH; 2011

11 Christlich Demokratische Union Deutschlands. Wachstum. Bildung. Zusammenhalt. Koalitionsvertrag zwischen CDU, CSU und FDP. 17. Legislaturperiode (26.10.2009). Im Internet: www.cdu.de Stand: 12.03 . 2011

12 Bewertungsausschuss Kassenärztliche Bundesvereinigung (KBV). Beschluss zur Änderung des Beschlusses des Bewertungsausschusses zur Neuregelung der Zuschläge für die Erbringung von ärztlichen Leistungen in Berufsausübungsgemeinschaften mit Wirkung zum 1.Juli 2011. Im Internet: www.kbv.de Stand: 19.05 .2011

13 Berges L. Zielgruppe MVZ. Pharma Marketing 2010; 6: 20-23

14 Armbruster S, Lubs S, Röhrig N, Wagner K. MVZ-Survey 2005. Eine Analyse der strategischen Positionierung Medizinischer Versorgungszentren. 1: Aufl. Berlin: KBV; 2006

15 Gibis B, Lubs S, Reuschenberg R, Schmidt C, Armbruster S. MVZ-Survey 2008. Die strategische Positionierung Medizinischer Versorgungszentren. 1: Aufl. Berlin: KBV; 2009

16 Robert Koch Institut. 20 Jahre nach dem Fall der Mauer: Wie hat sich die Gesundheit in Deutschland entwickelt? Beiträge zur Gesundheitsberichterstattung des Bundes. 1. Aufl. Berlin: RKI; 2009

17 Kassenärztliche Bundesvereinigung (KBV). Medizinische Versorgungszentren aktuell 2.Quartal 2010 (30.06.2010). Im Internet: www.kbv. de Stand: 14.03 .2011

18 Kassenärztliche Bundesvereinigung (KBV). Medizinische Versorgungszentren aktuell 4.Quartal 2010 (31.12.2010). Im Internet: www.kbv. de Stand: 16.09.2011

19 Gibis B, Köhler A. Ambulante Chirurgie in Medizinischen Versorgungszentren: Trends und Entwicklungen. Zentralbl Chir 2011; 136: 185189

20 Kassenärztliche Bundesvereinigung (KBV). Entwicklung der Medizinischen Versorgungszentren (31.12.2010). Im Internet: www.kbv.de Stand: 16.09.2011

21 Grote Westrick M. Versorgungsqualität durch Koordination und Kooperation steigern. In: Grote Westrick M, Schwenk U. Qualität in integrierten Versorgungstrukturen. Qualitätsindikatoren für Medizinische Versorgungszentren. 1. Aufl. Gütersloh: Bertelsmann Stiftung; 2010

22 Scheuerlein H, Settmacher U. Gedanken zur Aus- und Weiterbildung zum Chirurgen - gestern, heute und morgen. Zentralbl Chir 2010; 135: $451-457$

23 Winzer K-J, Bellach J. Wertigkeit der routinemäßig erfassten Nachsorgedaten bei Brustkrebspatientinnen. Zentralbl Chir 2010; 135: 257-261

24 Schreiner M, Spazier M, Wayand W. Diagnostik der akuten Appendizitis im Verlauf von 2 Jahrzenten - Auswirkungen der bildgebenden Verfahren auf präoperative Diagnosesicherung, Kosten und Patientenoutcome. Zentralbl Chir 2010; 135: 336-339

25 Pfister K, Krammer S, Janotta $M$ et al. Welche Nachkontrolle ist bei einer endovaskulären Versorgung von abdominellen Aortenaneurysmen empfehlenswert? Zentralbl Chir 2010; 135: 409-415

26 Köppl B. Pressemitteilung BMVZ vom 1. November 2010. Berlin: BMVZ; 2010 